\title{
Untersuchungen über die Ausbreitung von Raumwellen
}

\author{
Von Walter Budde* \\ (Z. Naturforschg. 5 a, 683-687 [1950]; eingegangen am 18. November 1950)
}

\begin{abstract}
Durch ein Zweibasissystem mit sehr kleinen inneren Fehlern werden erhebungswinkelunabhängige Azimutbestimmungen und Messungen der Großkreisausbrechungen von Kurzwellen, die sich über die Ionosphäre ausbreiten (Raumwellen) möglich. Neben rasch verlaufenden Schwankungen wird ein täglicher Gang der Großkreisausbrechungen festgestellt, der auf die Veränderung der Schräglage der Ionosphäre durch die Sonneneinstrahlung zurückgeführt wird.
\end{abstract}

$\mathrm{D}^{\mathrm{i}}$ ie Großkreisausbrechungen (Abweichungen vom Großkreis Sender - Empfänger) von Wellen, die an der Ionosphäre reflektiert werden, und ihre Schwankungen stellen die Grenze der Genauigkeit der Richtungsmessung mit Ionosphärenwellen dar. Für die Funkpeilung sind daher Messungen der Großkreisausbrechungen und ihrer Schwankungen von großer praktischer Bedeutung. Wichtig ist die Bestimmung der Großkreisausbrechungen weiterhin für eine zweckmäßige Gestaltung von Sende- und Empfangsanlagen, die dem kommerziellen Verkehr über große Entfernungen dienen und die man vorzugsweise mit Richtcharakteristik ausbildet, um die Störgeräusche niedrig zu halten. Schließlich tragen die Messungen der Großkreisausbrechungen in starkem Maße dazu bei, unsere Kenntnisse über die Struktur der Ionosphäre und ihre zeitliche Änderung zu vertiefen.

Bei diesen Messungen stößt man jedoch auf Schwierigkeiten, insbesondere dann, wenn es sich um Messungen an einer kontinuierlichen Strahlung handelt. Wird eine Raumwelle auf ihrem Weg vom Sender zum Empfänger an der Ionosphäre reflektiert, so ist es möglich, daß sie eine Drehung ihrer Polarisationsebene erleidet. Diese Drehung der Polarisationsebene kann zu einer Verfälschung des Ergebnisses führen, wenn man das Einfallsazimut der Welle am Empfangsort bestimmt (Nachteffekt). Bei manchen Verfahren der Richtungsmessung ist der gemessene Wert des Azimuts noch abhängig von dem Erhebungswinkel, unter dem die Welle am Empfangs-

* Aachen, Karl-Marx-Allee 154.

1 P. Kotowski, E. S ch üttlöffel u. G.Vogt, Arch. elektr. Übertragung 4, 325 [1950].

2 P. v. H a nd el, Über die Grenzen der Genauigkeit der Richtungs- und Entfernungsmessung mit drahtlosen Wellen über große Strecken. Deutsch. Akad. Luftfahrtforschg., Juli 1942; Großbasisverfahren "Komet”, DVLBericht 1942; Elektron 1, 366 [1947]. ort einfällt. Von A d c ock wurde im Jahre 1918 ein Verfahren angegeben, daß sowohl den Polarisationsfehler als auch den Erhebungswinkelfehler bei der Azimutbestimmung ausschaltet. Jedoch können auch bei der Adcock-Anlage ganz beträchtliche Fehler auftreten. Diese Fehler sind darauf zurückzuführen, daß manchmal die Strahlung auf mehreren Wegen gleichzeitig vom Sender zum Empfänger gelangt. Von Kot owski, Schüt t löffel und Vogt wurden bis zu 5 gleichzeitig vorhandene Ausbreitungswege festgestellt ${ }^{1}$. Es sind dann am Empfangsort zur gleichen Zeit mehrere, meist nahe beieinander liegende Einfallsazimute vorhanden. Die Adcock-Anlage zeigt in diesem Fall im allgemeinen jedoch nicht etwa einen Mittelwert an, sondern es können ganz abwegige Azimutwerte vorgetäuscht werden.

Durch theoretische Untersuchungen konnte v. $\mathrm{H}$ andel nachweisen, daß bei Mehrwegeausbreitung die Fehlweisung der Adcock-Anlage den wahren Wert unter Umständen um ein Vielfaches übertreffen kann $^{2}$. Diese Schwierigkeiten bei der Azimutbestimmung lassen sich dadurch umgehen, daß man vom Betrieb mit kontinuierlicher Strahlung zum Impulsbetrieb übergeht und dadurch in die Lage kommt, die einzelnen Wege trennen zu können. Von v. Han • del und Crone konnte weiterhin gezeigt werden, daß auch bei kontinuierlicher Strahlung die durch Mehrwegeausbreitung entstehenden Mißweisungen kleiner werden, wenn man die Basis des richtungsempfindlichen Systems vergrößert. Von $\mathrm{Cr}$ on $\mathrm{e}^{3}$ und Martin wurde eine Anlage erstellt mit $d / \lambda=2$; $d=$ Basis, $\lambda=$ Wellenlänge. (Das Adcock-System hat einen sehr viel kleineren Wert von $d / \lambda$.) Die Versuche zeigten in Übereinstimmung mit der Theorie, daß in

3 W. Cro n e, Möglichkeiten und Grenzen der Richtungsmessung mit Raumwellen; Beschreibung der Anlage „Komet“, Berichte des Ferdinand-Braun-Institutes Landsberg a. Lech [1944]. 


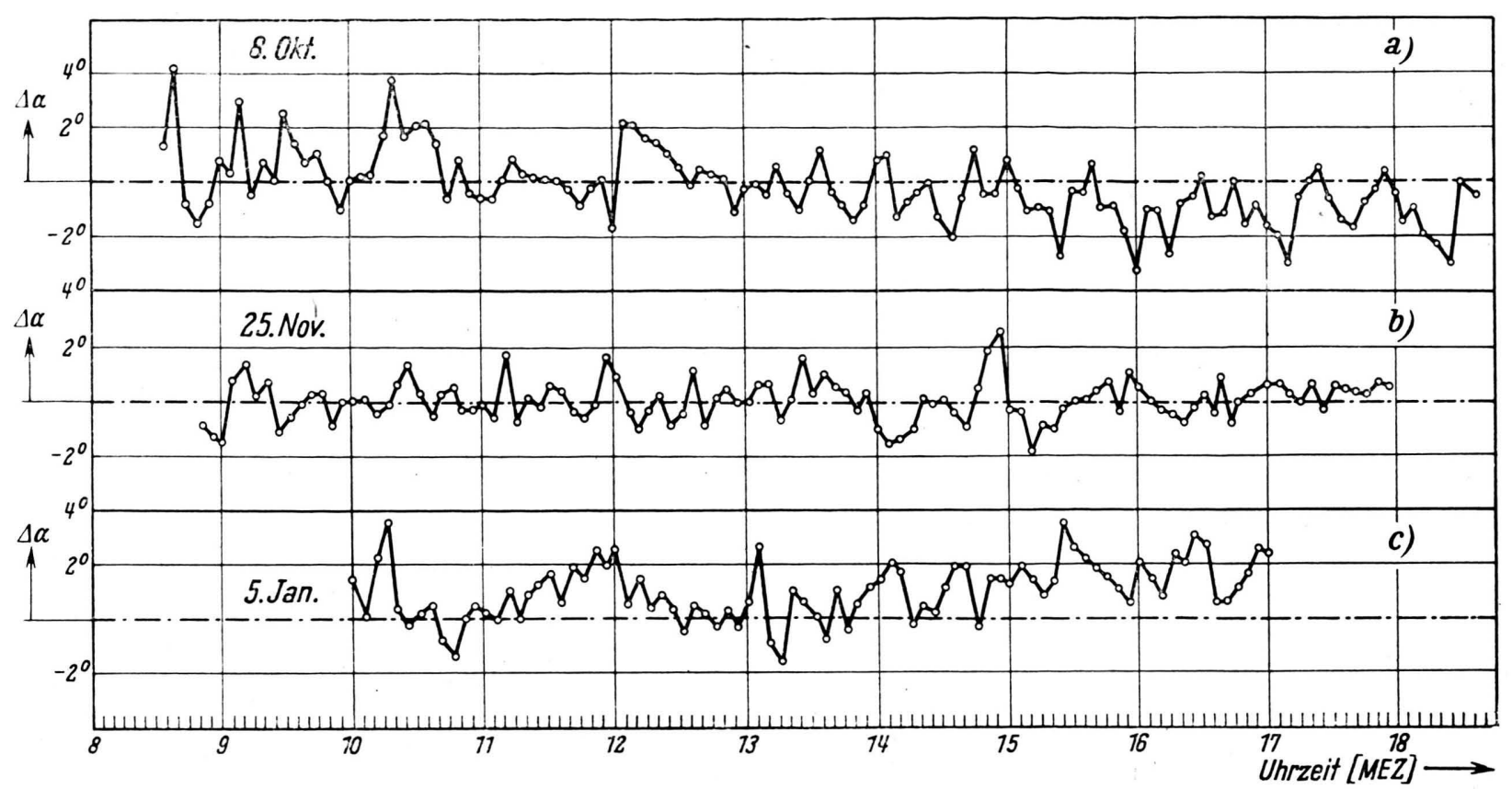

Abb. 1. Großkreisausbrechungen in Abhängigkeit von der Tageszeit. ------ geometrischer Wert des Azimuts.

diesem Fall die Fehlweisungen vernachlässigbar klein werden. Der Wert $d / \lambda=2$ durfte nicht überschritten werden, wenn der Empfang von kohärenten Wellen durch alle Antennen des Systems gewährleistet bleiben sollte.

Mit einer solchen Einbasisanlage wurden jahrelang Versuche zwischen Templin/Uckermark (Sender) und verschiedenen Stellen in Italien und Nordafrika (Empfänger) gemacht, die ein reiches Beobachtungsmaterial über die Ausbreitung der Kurzwellen ergaben ${ }^{3}$.

Die erstellte Anlage war jedoch nur für einen verhältnismäßig kleinen Winkelbereich, innerhalb dessen auch die Messungen durchgeführt wurden, frei von Erhebungswinkelfehlern. Eine für jeden Wert des Azimuts von Erhebungswinkelfehlern freie Richtungsbestimmung wurde durch Zugrundelegung eines anderen Meßprinzips bei einer in Ismaning b. München erstellten Zweibasisanlage erreicht ${ }^{4}$.

Die einfachste Methode der Azimutbestimmung ist die, ein richtungsempfindliches System mechanisch zu drehen. Jedoch sind diese Systeme sehr schwerfällig und gestatten nur recht ungenaue Messungen. Vor allem aber sind sie ganz ungeeignet dazu, schnell verlaufende Schwankungen des Einfallsazimuts zu verfolgen. Die heute fast nur noch vorkommenden

4 W. B u d d e, Diss. T. H. München 1947, Arch. elektr. Ühertragung (im Druck); Z. Naturforschg. 5a, 291 [1950].
Anlagen mit elektrisch schwenkbarer Charakteristik beruhen im Prinzip darauf, daß die Phasendifferenzen zwischen den in den Antennen des Systems fließenden Strömen von der Richtung der einfallenden Strahlung abhängen bzw. die Richtung des Maximums oder Minimums der von dem System weggehenden Strahlung bestimmen.

Das verwendete richtungsempfindliche System erzeugte ein Strahlungsfeld, das dadurch gekennzeichnet war, daß in ihm Leitstrahlflächen entstanden, auf denen eine Modulation von $70 \mathrm{~Hz}$ verschwand, die im übrigen Strahlungsfeld vorhanden war. Durch Drehung der Phasen der Antennenströme wurden diese Leitstrahlflächen durch den Raum geschwenkt. Am Empfangsort wurden diejenigen Zeitpunkte registriert, zu denen die 70-Hz-Modulation verschwand. So ließ sich dasjenige Azimut bestimmen, unter dem die Strahlung, die den Empfangsort erreichte, den Sender verließ. Es wurde also nicht das Einfallsazimut am Empfangsort, wie in ${ }^{1}$, sondern das Ausfallsazimut am Sender bestimmt.

\section{Meßergebnisse}

Die folgenden Meßergebnisse wurden in der Zeit vom 8. Oktober 1944 bis 15. Januar 1945 auf der Strecke Ismaning/München (Sender) - Koelby/Däne- 


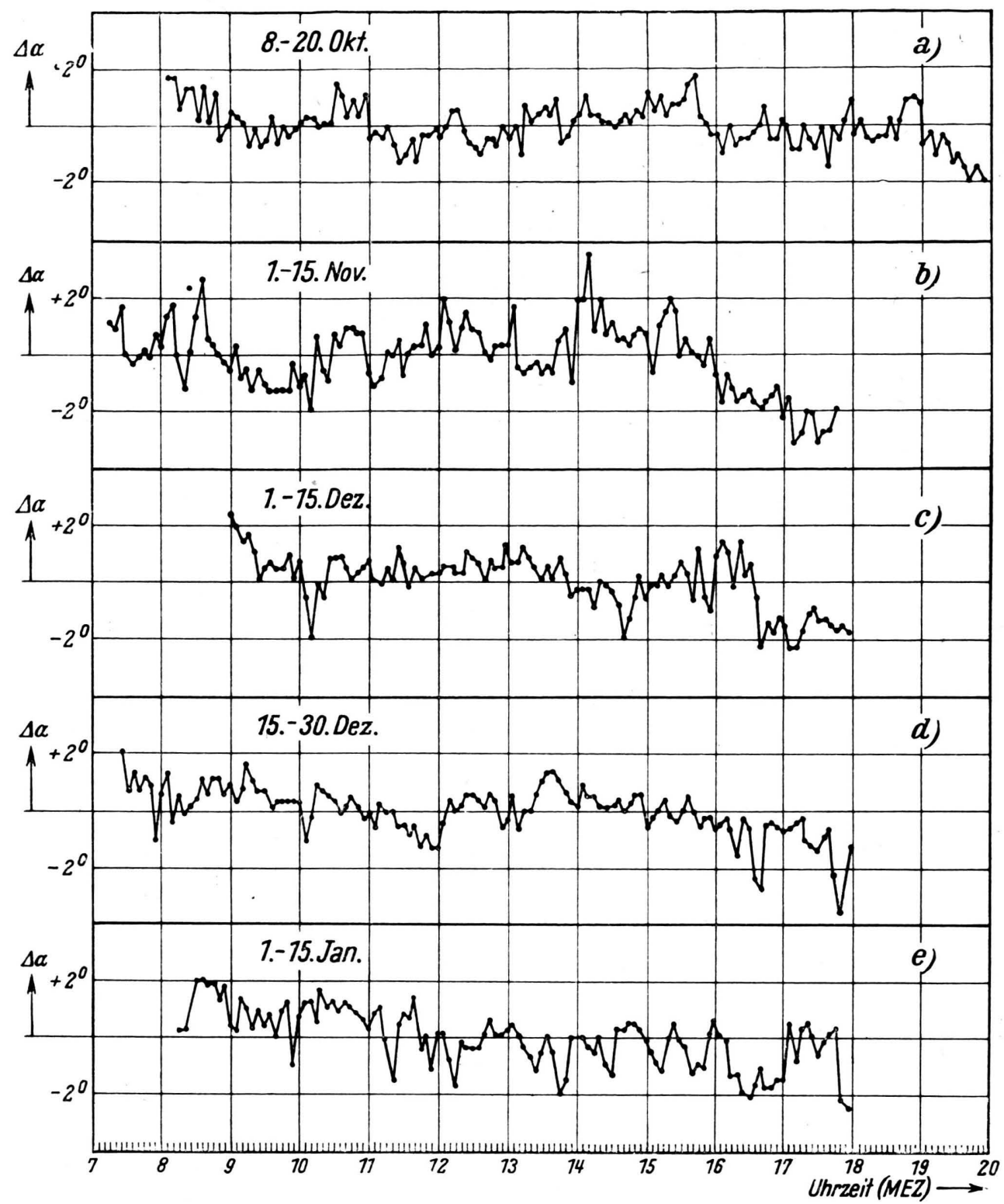

Abb. 2. Mittelwert der Großkreisausbrechungen in Abhängigkeit von der Tageszeit.

mark (Empfangsort) mit einem Großbasissystem $(d / \lambda=2)$ gewonnen. Die verwendete Frequenz betrug $9830 \mathrm{kHz}$.

Abb. 1 a zeigt für einen Oktobertag die Abhängigkeit des Ausfallsazimutes von der Zeit. Als Abszisse ist die Uhrzeit in MEZ aufgetragen. Als Ordinate ist aufgetragen die Abweichung $\Delta \alpha$ des Ausfallsazimuts rom geometrischen Wert des Azimuts, der kenntlich gemacht ist. Weicht die ausfallende Strahlung nach Osten von der Großkreisrichtung (dem geometrischen Wert des Azimuts) ab, so ist dies durch einen posi- tiven Wert von $\Delta \alpha$ gekennzeichnet; weicht sie nach Westen ab, so entspricht dem ein negativer Wert von $\Delta \alpha$. Die einzelnen Meßpunkte stellen dabei den zeitlichen Mittelwert über je 5 Min. dar.

In Abb. 1 a erkennt man das Schwanken des Ausfallsazimuts um die Großkreisrichtung. Vor allem fällt auf, daß neben verhältnismäßig kurzzeitigen Schwankungen ein über die Zeit vom Morgen bis zum Abend sich erstreckender Gang der Großkreisausbrechungen $\mathrm{zu}$ erkennen ist. Am Morgen wird vorzugsweise die etwas östlich vom Großkreis ausfallende 
Strahlung vom Empfänger aufgenommen. Während der Mittagszeit ist keine systematische Abweichung vom Großkreis zu erkennen. Mit fortschreitender Tageszeit nimmt der Empfänger in immer stärker werdendem Maße diejenige Strahlung auf, die in einer Richtung westlich der Großkreisebene den Sender verläßt. Auch $\mathrm{Cr}$ one und Weber stellten bei den Messungen mit dem Einbasissystem einen Gang des Mittelwertes des Ausstrahlungsazimuts fest ${ }^{3}$. Die Messungen von Kotowski, Schüttlöffel und Vog t ließen ebenfalls neben schnellen, oft sprunghaften Änderungen auch langsame Wanderungen des Mittelwertes erkennen ${ }^{1}$. Bei den Messungen mit der Zweibasisanlage war jedoch nicht an allen Tagen dieser Gang zu erkennen, wie Abb. $1 \mathrm{~b}$ zeigt. Hier hat der Mittelwert des Ausfallsazimuts den gुanzen Tag über den gleichen Wert. Manchmal wurde auch der umgekehrte Effekt gefunden, daß nämlich die positive Abweichung mit der Zeit langsam anstieg, wie Abb. 1c für die Zeit zwischen $12^{00}$ und $16^{00} \mathrm{Uhr}$ zeigt. An manchen Tagen war der Mittelwert der positiven Abweichungen morgens besonders groß, sank dann aber im Verlauf von 1-2 Stdn. auf den geometrischen Wert.

Einen guten Überblick erhält man, wenn man über die Messungen mehrerer Tage mittelt. Abb. 2 a zeigt die Mittelwerte der zwischen dem 8. und dem 20. Oktober gemachten Messungen. Als Abszisse ist wieder die Uhrzeit in MEZ aufgetragen. Als Ordinate ist aufgetragen das arithmetische Mittel aus denjenigen Meßwerten, die an verschiedenen Tagen um die gleiche Uhrzeit aufgenommen wurden, bezogen auf den halbmonatlichen Mittelwert des gemessenen Azimuts. Dabei ist jedoch das Mittel nur von denjenigen Werten genommen, bei denen der Empfang einwandfrei war. Man bemerkt vor allem den steilen Abfall der Mittelwerte von großen positiven Abweichungen morgens nach Beginn der Registrierungen. Während des Tages schwanken die Meßwerte um den geometrischen Wert. Am Abend fallen die Mittelwerte weiter steil zu negativen Abweichungen ab.

Abb. 2 b zeigt eine der Abb. 2 a entsprechende Darstellung für die erste Hälfte des Novembers. Auch hier erkennt man deutlich die großen positiven $\mathrm{Ab}$ weichungen sowie den schnellen Abfall zum Großkreiswert in den Morgenstunden und weiterhin den Abfall zu verhältnismäßig großen negativen Abweichungen am Abend. Der Abfall am Abend erstreckt sich über eine längere Zeit und setzt auch, deutlich erkennbar, früher ein als in Abb. 2 a. Die Abb. 2c und $2 \mathrm{~d}$ zeigen die Mittelwerte der in der Zeit vom 1. bis 15. Dezember bzw. vom 15. bis 30. Dezember gewonnenen Meßergebnisse. Auch hier ist der Abfall in den Morgen- und Abendstunden deutlich zu erkennen.

Die Mittelwerte der in der ersten Januarhälfte gemachten Messungen zeigt Abb. 2 c. Hier fällt besonders der langsame, aber stetige Abfall der Werte während des ganzen Tages auf, wie er bereits an der Einzelregistrierung von Abb. 1 a gezeigt wurde. Gegen $16^{00}$ Uhr beginnt ein verstärkter Abfall, dem jedoch ein erneuter Anstieg folgt.

Die systematischen Großkreisausbrechungen morgens und abends und ihr zeitlicher Verlauf wurden auch bei den Messungen mit der Einbasisanlage festgestellt und von Crone und Weber folgendermaßen gedeutet: Bei Sonnenaufgang erhält der Gradient der Trägerdichte in der Ionosphäre eine Komponente in west-östlicher Richtung. Dies wirkt sich aus wie eine Schräglage der Ionosphäre, und zwar scheint bei Sonnenaufgang die Ionosphäre im Osten tiefer, im Westen höher zu liegen. Strahlen, die den Sender in nördlicher Richtung verlassen, werden also bei ihrer Reflexion an der Ionosphäre nach Westen abgelenkt. Diejenigen Strahlen, die den Sender in Richtung des Großkreises Sender-Empfangsort nach Norden verlassen, erreichen den Empfänger nicht. Die Strahlen, die den Empfangsort erreichen, verlassen den Sender in einer Richtung östlich der Großkreisrichtung. Analoge Betrachtungen für den Abend zeigen, daß dann die Ionosphäre im Westen tiefer, im Osten höher ist. Das Aúsfallsazimut der den Empfänger erreichenden Strahlung liegt dann westlich der Großkreisrichtung.

Ist $h$ die Höhe der Ionosphäre, $\Delta \varphi$ der Winkel zwischen der Ionosphäre und der Horizontalen in westöstlicher Richtung, $l$ die Entfernung Sender-Empfänger, so ergibt sich für die Großkreisausbrechung $\Delta \alpha$ für kleine Winkel und unter der Annahme nur einmaliger Reflexion der Strahlung an der Ionosphäre:

$$
د \iota=\frac{2 h \Delta \varphi}{l} .
$$

Man sieht, daß die Großkreisausbrechungen mit zunehmender Entfernung des Empfängers vom Sender abnehmen. Dies stellten auch Kotowski, Schüttlöffel und Vogt fest, die fanden, daß die Schwankungen der Großkreisausbrechungen bei nahen englischen Kurzwellensendern bedeutend größer waren als bei den weiter entfernten amerikani- 
schen. Weiterhin sieht man, daß die Großkreisausbrechungen linear mit der Höhe der reflektierenden Schicht zunehmen.

Untersuchungen über die Genauigkeit der Zweibasisanlage, wenn diese als System für Richtungs-

5 R. W e b e r, Bericht des Ferdinand-Braun-Institutes Landsberg a. Lech Nr. 27 [1945]. messungen benutzt wird, wurden von $\mathrm{R}$. Web er angestellt ${ }^{5}$.

Den HHrn. Prof. Dr.-Ing. Paul Frhr. v. Handel, Dr. phil. Gerh ard Martin, Dr.-Ing. Wilh. Crone, Studienrat Rudolf Weber, Dipl.-Ing. Erich Humm rich und Dr. rer. nat. Alfons Friedel bin ich für die Übertragung der Arbeit und ihre Hilfe bei der Durchführung zu großem Dank verpflichtet.

\section{NOTIZEN}

\section{${ }^{84}$ Rubidium, ein neues Isomer von $T=23 \mathrm{~min}$ Halbwertszeit}

Von A. F la m mersfeld

Max-Planck-Institut für Chemie, Mainz

(Z. Naturforschg. 5 a, 687-688 [1950]; eingeg. am 27. Nov. 1950)

Beim Rubidium sind 3 Aktivitäten bekannt, die bei Bestrahlung mit Neutronen auftreten: ${ }^{88} \mathrm{Rb}(T=17,8 \mathrm{~min})$, ${ }^{86} \mathrm{Rb}(T=19,5 \mathrm{~d})$ und ${ }^{84} \mathrm{Rb}(\approx 40 \mathrm{~d})$, die jeweils durch ${ }^{87} \mathrm{Rb}(\mathrm{n}, \gamma) 1,{ }^{85 R b}(\mathrm{n}, \gamma) 2$ und $85 \mathrm{Rb}(\mathrm{n}, 2 \mathrm{n}) 3$ erhalten worden sind.

In der vorliegenden Arbeit sollten die durch (n, 2n)Prozeß entstehenden Aktivitäten untersucht werden, wozu Präparate von sehr reinen Rubidiumsalzen $\left(5-7 \mathrm{mg} / \mathrm{cm}^{2}\right.$ ) auf einer dünnen Kohleunterlage mit Li+D-Neutronen $\left(E_{\mathrm{D}}=1,4 \mathrm{MeV}\right)$ bestrahlt und in einem Schleusenzählrohr (Fenster ${ }^{1} / 2 \mu$ Zaponlack) gemessen wurden. Hierbei zeigte sich außer den bekannten Halbwertszeiten noch eine bisher unbekannte von $T=23 \mathrm{~min}$, die zwar mit beträchtlicher Intensität auftritt, aber wegen der geringen Energie der ausgesandten Elektronen und der mit ${ }^{88} \mathrm{Rb}$ sehr ähnlichen Halbwertszeit offenbar bisher der Beobachtung entgangen ist.

Abb. 1 gibt die Versuchsergebnisse. Kurve A und B stellen den zeitlichen Abfall eines mit verlangsamten $\mathrm{Be}+\mathrm{D}-$ Neutronen bestrahlten Rubidiums dar, und zwar Kurve A ohne und Kurve B mit einem Absorber von $100 u$ Aluminium vor dem Zählrohr. In Übereinstimmung mit der großen $\beta$-Energie des ${ }^{88} \mathrm{Rb}$ ist die Absorptionswirkung von $100 \mu \mathrm{Al}$ zu Anfang der Kurven, wo der 17,8-minKörper dominiert, recht gering.

Die entsprechenden Kurven für mit Li+D-Neutronen bestrahltes Rubidium sind Kurve C ohne und D mit $100 \mu$ Aluminiumabsorber. Sie zeigen erstens eine langlebige Komponente, die wahrscheinlich aus einem Gemisch von ${ }^{86} \mathrm{Rb}$ und ${ }^{84} \mathrm{Rb}$ besteht, welche durch $(\mathrm{n}, 2 \mathrm{n})$ und $(\mathrm{n}, \gamma)$ Prozesse aus den stabilen Isotopen entstanden sind, und zweitens eine kurzlebige Komponente, die eine wesentlich geringere Energie als ${ }^{88 \mathrm{Rb}}$ besitzt, wie sich z. B. aus der

1 E. Fermi, E. Amaldi, O. D'Agostino, F. R a s e t ti u. E. S e grè, Proc. Roy. Soc. [London], Ser. A 146, 483 [1934].

2 A. H. S n e l 1, Physic. Rev. 52, 1007 [1937]; H. S c h e i c h e n b e r g e r, Anz. Akad. Wiss. Wien, math.-naturwiss. Kl. Nr. 17 [1938].

3 W. C. B a r b e r, Physik. Rev. 72, 1156 [1947]. erheblich größeren Differenz der Kurven C und D ergibt, und wie es eine vollständige Absorptionskurve genauer zeigt.

Diese neue Aktivität tritt nur mit Li+D-Neutronen auf, schnelle $\mathrm{Be}+\mathrm{D}$ - oder verlangsamte Neutronen erzeugen sie nicht. Da aber bei Versuchen mit schnellen Neutronen sich $(\mathrm{n}, \gamma)$-Prozesse nicht vollkommen vermeiden lassen, muß wohl auch hier angenommen werden, daß die kürzerlebige Komponente der Kurve $\mathrm{C}$ eine Mischung der alten 17,8-min-Substanz mit der neuen darstellt, so daß deren Untersuchung stark erschwert wird.

Die Halbwertszeit wird daher am besten aus der Differenz der kurzen Komponenten der Kurven C und D ermittelt, welche als Kurve $\mathrm{E}$ eingezeichnet ist und für die neue Aktivität $T=23 \pm 1 \mathrm{~min}$ ergibt. Aus vollständigen Absorptionsmessungen kann geschätzt werden, daß der Beitrag der 17,8-min-Aktivität zur Kurve C höchstens $20 \%$

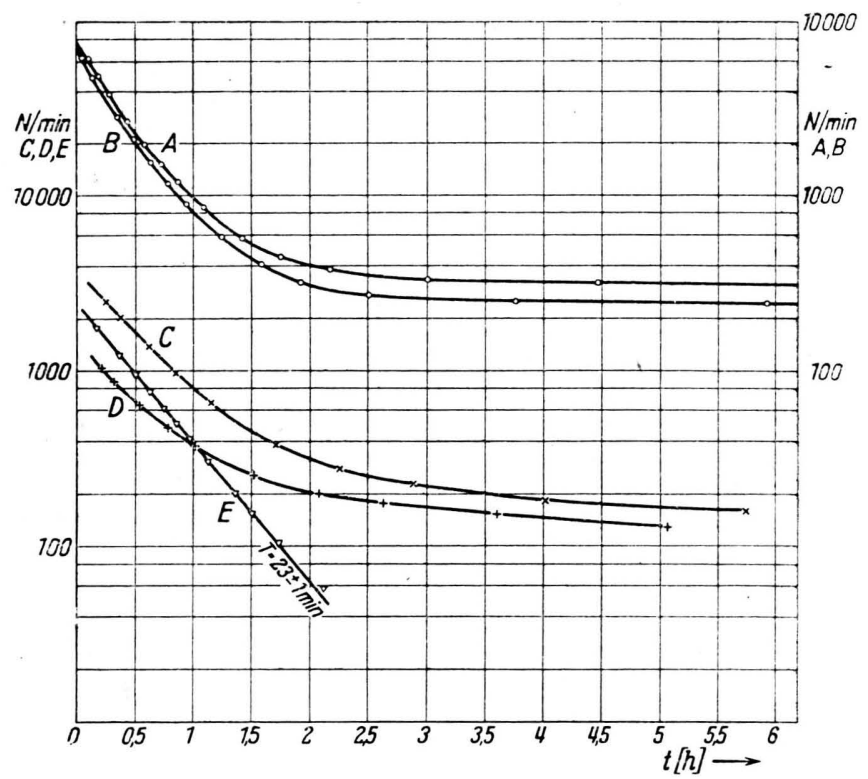

Abb. 1. Kurven A und B: Zeitlicher Abfall eines mit langsamen Neutronen bestrahlten Rubidiums. A ohne, B mit $100 \mu \mathrm{Al}$-Absorber vor dem Zähler. Kurven C und D: dasselbe mit schnellen Li+D-Neutronen. C ohne, D mit $100 \mu$ Al-Absorber. $\mathrm{E}=$ Differenz der kurzlebigen Komponenten von $\mathrm{C}$ und $\mathrm{D}$. 